\title{
23
}

\section{Towards Design Formalization to Support Reuse in ECAD Frameworks}

\author{
Joachim Altmeyer, Bernd Schuermann, Martin Schuetze \\ University of Kaiserslautern, Germany
}

\begin{abstract}
'Design Reuse' is one of the hopeful techniques to increase the productivity in ECAD frameworks. To support this reuse we propose a formal model to describe design objects, design processes, and requirement specifications. Using this model we introduce a measure to determine reuse candidates from the design database. We address the consideration of special CAD properties and discuss appearing problems.
\end{abstract}

\section{Introduction}

The reuse of designs or parts of designs seems to be "the key enabler for system design in the 90's" [DAC94]. This reuse should comprehend all design informations: design objects as well as specifications, documentations, activities, and whole design processes. To turn this effort into reality we need an adequate formalization of the whole design. Even if our goal must be to support the reuse of all design data, we will mainly focus on the reuse of design objects and design specifications in this paper.

In section 2, we gather the requirements of a comprehensive reuse in ECAD frameworks. Section 3 focuses on the formalization of designs to support the retrieval of suitable reuse candidates. We address important problems and we investigate which of these problems can be solved by our model. In section 4 , we discuss the application of this model and we present a survey of interesting current and future works.

\section{Reuse in ECAD}

To date, reuse is only supported rudimentarily in existing ECAD frameworks. It is restricted to the utilization of standards (e.g. the IEEE floating-point standard) or building generic descriptions of design objects or processes (e.g. generic nets in [BHK87]) [GiC93].

Let us collect properties which distinguish reuse in $\mathrm{CAD}$, especially in $\mathrm{ECAD}$, from many other reuse situations: 


\section{$\square$ Design Space}

Design objects in the design database are part of dependency networks (see figure 1):

First, design objects are often aggregates of other design objects (part-of hierarchy). Second, every object has a history which expresses its evolution (design hierarchy). Third, there are similarities of independently designed objects defined by their properties (property or feature network). For instance, some layouts have the same technology or they concern to the same library of arithmetical functions fulfilling the IEEE floating point standard.

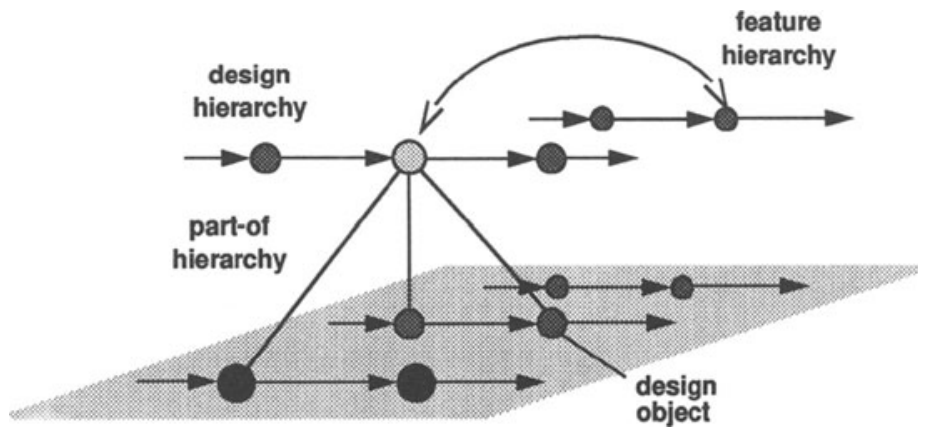

Figure 1: Design Space

\section{$\square$ Complex Objects}

Design objects are complex objects in the sense that they own non atomic attributes, e.g. a netlist or a whole layout description.

\section{$\square$ Requirement Specification}

Every design is initiated by a requirement specification which must be described in an adequate form.

$\square$ Design Process

Design processes are complex processes (e.g. the processes are nested).

\section{$\square$ Design Environment}

To describe a design in its entirety a lot of related documents is necessary (e.g. a document that describes the role of the involved tools or designers [CFI91], [HRS92]). In addition to this, there is a lot of knowledge which is not available by computers (e.g. the designers knowledge).

\section{Amount of Data}

The amount of data in ECAD is immense. 
We can distinguish different reuse methods. Reuse can be realized by instantiation, by parameterization (generalization), or by adaptation [GiC93] [AOS94]. At reuse by instantiation, the reuse of optimized and validated objects is supported by a standardization of these. Then, these objects become types and can be instantiated within new designs. One precondition is that the underlying data model supports instantiation. For this, many suitable data models were proposed at earlier ECAD conferences. At reuse by instantiation, the creative part is to find suitable components and to integrate them well into the current design.

Furthermore, it is possible to describe components generically. For instance, we can describe an adder with the bit-width as a formal parameter. We call this reuse by generalization or parameterization. Here, the creative part is to generalize the description of a component by creating formal parameters.

In the last case, already designed solutions are reused by adapting these to the current design situation. At this reuse by adaptation, it is the goal to retrieve suitable components and to support the designer for adapting these components. To find suitable components means that the found solutions must fit the given requirement specification best. In contrast to the reuse by instantiation, the found components are then fitted to their environment not vice versa. This reuse process is outlined in figure 2. In this paper, we focus on the description of design objects, requirement specifications and design processes to support the identification of suitable reuse candidates.

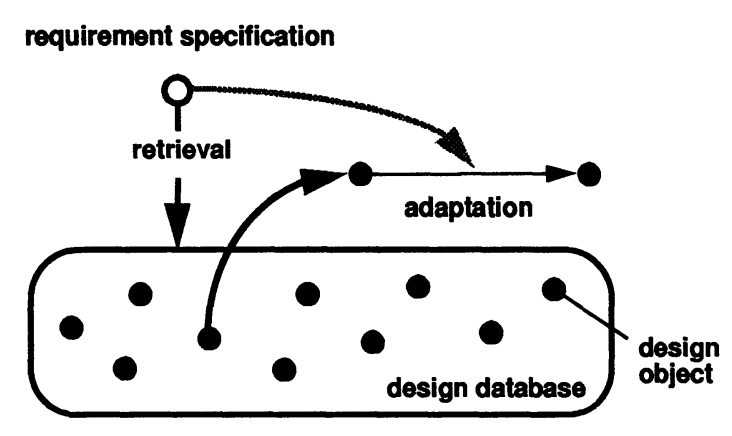

Figure 2: Reuse by Adaptation

\section{Reuse Model}

To support reuse in ECAD regarding the properties of the previous section we need an adequate formalization of the design, especially of the design objects, specifications, and design processes. First design formalizations without considering reuse can be found in [Yos81], [Ger90], 
or [Sie91]. In this section, we present the formalization of our feature-based reuse model in detail. In a first step, we focus on the description of design objects, requirement specifications, and processes.

To describe design objects and specifications we propose two definitions:

Definition 1 (feature or property)

$\mathrm{x}$ is a design object or a design specification. $\mathrm{p}$ is a feature (property)

of $x$ iff $p$ is a predicate with $p(x)$ is true.

\section{Definition 2 (feature set)}

The feature set of a design object or a design specification $\mathbf{x}$ is defined as $P_{x}=\{p \mid p(x)\}$.

These features do not depend on the data representation of the object. Our feature notion is a generalization of the notion 'feature' or 'form-feature' in geometric $\mathrm{CAD}$. The set of all possible features is divided into some clusters which we call feature classes. This helps us to organize features concerning the same domain. Examples of feature classes are function, bit-width, arity, technology, and area.

We extended the definition 1 and 2 from design objects to requirement specifications because even if there is a difference between specifications and design objects in their role within the design process, there is no difference in their characterization using feature sets.

In every feature class, a generalization-specialization relation ' $<$ ' is defined. For instance, a feature 'Arity is 4' is a specialization of a feature 'Arity is Even', i.e. 'Arity is 4' < 'Arity is Even'. Using this relation the instances of each feature class builds a mathematical lattice structure with the least element 'no specification' and a greatest element which spans the whole definition space of the feature class [OnY87].

Now, we define generic features:

Definition 3 (generic features)

A generic feature $g$ defines a set $G$ of features with $G=\{p \mid p<g\}$

where $p$ is a feature.

Using this definition we are able to handle a generic feature $g$ within a feature set $P_{i}$ in the way that we replace it by the features of the corresponding set $\mathrm{G}$ which results in a more simple and uniform model:

$$
P_{i}^{\prime}=\left(P_{i}-\{g\}\right) \cup G
$$

Before we define similarity functions between design objects and specifications, we describe the correlations between the definitions above and the design process: 
Definition 4 (successful design)

$\mathbf{s}$ is a design specification. The design is successful iff there exists a design object $\mathrm{x}$ with $\mathrm{P}_{\mathrm{S}} \subseteq \mathrm{P}_{\mathrm{X}}$.

Definition 5 (refinement design step)

$\mathrm{x}$ and $\mathrm{y}$ are design objects and $\mathrm{s}$ is a design specification. $\varphi$ is a refinement design step iff $\varphi(x)=y$ and $\left(\mathrm{P}_{\mathrm{S}} \cap \mathrm{P}_{\mathrm{x}}\right) \subseteq\left(\mathrm{P}_{\mathrm{S}} \cap \mathrm{P}_{\mathrm{y}}\right)$.

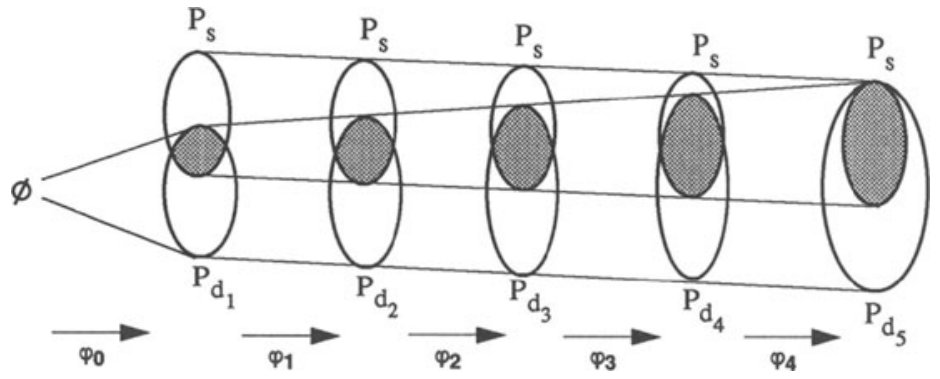

Figure 3: Venn Diagrams of Design Refinement Steps $P_{S}$ is the feature set of the overall requirement specification $s, \varphi_{i}$ are refinement steps, $P_{d_{i}}$ are the feature sets of the design objects $d_{i}$

Or with other words: "With refinement steps, we come closer to fulfil the overall design specification". Figure 3 illustrates the evolution process. These refinement steps define the design hierarchy which describes the evolution of the design. Every object, which results from a refinement design step, can be reused by itself. This is the reason why we call every object resulting from a refinement step a design object. It is important that, during the design, there are other steps, e.g correction steps or analyzing steps.

\section{Example 1 (design hierarchy)}

In figure 4, the design hierarchy is illustrated by an example using the PLAYOUT design system [Zim89]. In a first step $\varphi_{0}$, a VHDL program $d_{1}$ was written from scratch $\left(d_{0}=\varnothing\right)$. In step $\varphi_{1}$, a high level synthesis tool was applied resulting with a netlist $d_{2}$ as a result. Then, two alternative floorplans $d_{3}$ and $d_{4}$ were designed. Using these, two layouts $d_{5}$ and $d_{6}$ were assembled. The overall design was successful regarding the requirement specification $\mathrm{s}$ iff $\mathrm{P}_{\mathrm{s}} \subseteq \mathrm{P}_{\mathrm{d}_{\mathrm{i}}}$ for a design object $d_{i} \in\left\{d_{1}, d_{2}, \ldots d_{6}\right\}$. 


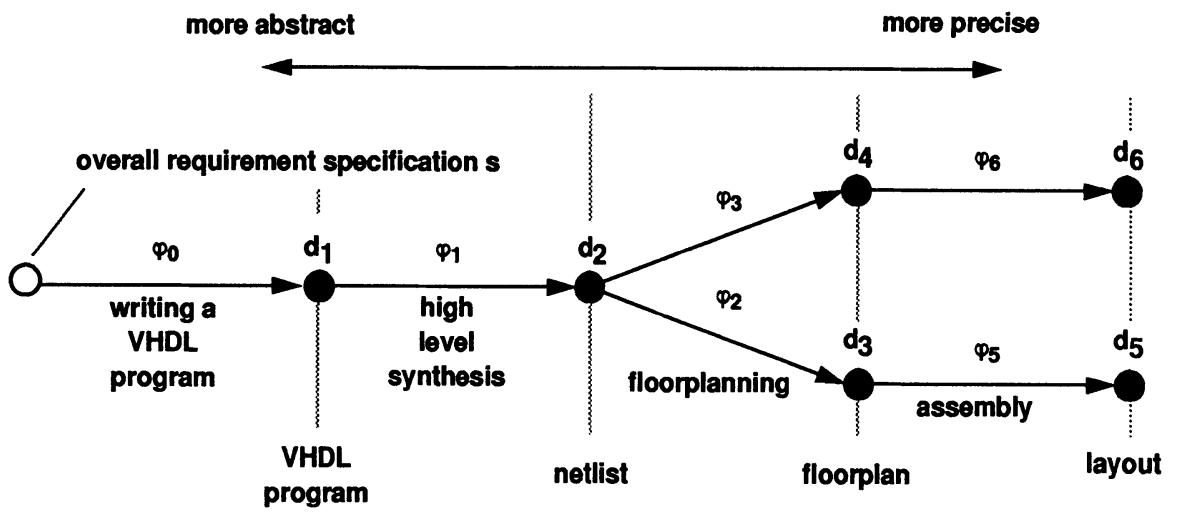

Figure 4: Example of a Design Hierarchy

So far, we introduced a characterization of design objects and specifications. In the following, we focus on their similarities. To determine the similarity of features we use individual (asymmetric) similarity functions for each feature class:

Definition 6 (feature similarity function)

$\mathrm{C}$ is a feature class. The feature similarity function $\operatorname{sim}_{\mathrm{C}}$ is defined as $\operatorname{sim}_{C}: C \times C \rightarrow[0,1]$. If $\operatorname{sim}_{C}(p, q)=1$, then the feature $p$ is equivalent to the feature $q$. In the case of $\operatorname{sim}_{C}(p, q)=0, p$ is totally different from q.

The feature similarity function $\operatorname{sim}_{C}(p, q)$ roughly expresses the expense of converting the feature $q$ to the feature $p$. We define a similarity function $\operatorname{SIM}_{C}$ for a feature class:

Definition 7 (feature similarity function of a feature class)

If the feature sets $P_{s}^{C}=\left\{p \mid p \in P_{s} \wedge p \in C\right\}$ and $P_{x}^{C}=\left\{p \mid p \in P_{x} \wedge\right.$ $p \in C$ \} contain only one element, the feature similarity function of the feature class $\mathrm{C}, \mathrm{SIM}_{\mathrm{C}}$, is $\operatorname{sim}_{\mathrm{C}}$ with the instances of $\mathrm{P}_{\mathrm{s}}^{\mathrm{C}}$ and $\mathrm{P}_{\mathrm{x}}^{\mathrm{C}}$, respectively. Otherwise, we use the following function:

$$
\operatorname{SIM}_{C}(s, x)=\frac{\sum_{p_{s} \in P_{s}^{C}} \max (\underbrace{\cup}_{p_{x} \in P_{x}^{C}}\left\{\operatorname{sim}_{C}\left(p_{s}, p_{x}\right)\right\})}{\left|P_{s}^{C}\right|}
$$

Now, we define a similarity function SIM for design objects which enables us to determine the reusability of design objects regarding a given specification: 
Definition 8 (reusability function)

$\mathrm{s}$ is a design specification, $\mathrm{x}$ a design object, and $\Gamma$ the set of all feature classes with instances in $\mathrm{P}_{\mathrm{s}}$. The reusability function SIM is defined as:

$$
\operatorname{SIM}(s, x)=\sum_{C \in \Gamma} r_{C} \cdot \operatorname{SIM}_{C}(s, x) .
$$

$\mathrm{r}_{\mathrm{C}} \in \mathbb{R}^{+}$are relevance factors (weight factors) which express the importance of the corresponding feature classes for the comparison (the sum of all weight factors has to be 1).

Using the extended function SIMC of definition 7, we are able to consider generic design objects, i.e. objects with generic features, as well as multi-functional design objects, i.e. objects with more than one feature at the feature class function.

Example 2 (multi-functional design object)

If we search for a multi-functional design object with specification $\mathrm{s}$ and $\mathrm{P}_{\mathrm{s}}=\{$ 'Function is Add', 'Function is Multiply', 'Width is 32', 'Arity is 2 ' $\}$ and we find a design object $x$ with the feature set \{'Function is Add', 'Width is 32', 'Arity is 2'\} then

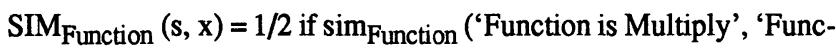
tion is Add') $=0$.

Regarding the definition of $P_{s}^{C}$ and $P_{x}^{C}$, resp., (see above) we define the context $P_{i}^{C}$ of a feature set as:

Definition 9 (context of a feature set)

$$
\overline{\mathrm{P}}_{\mathrm{i}}^{\mathrm{C}}=\mathrm{P}_{\mathrm{i}}-\mathrm{P}_{\mathrm{i}}^{\mathrm{C}} \text { is the context of } \mathrm{P}_{\mathrm{i}}^{\mathrm{C}} \text {. }
$$

One of the problems with the formula of definition 8 is that each feature set $P_{s}^{C}$ and $P_{x}^{C}$, resp., is investigated independently of its context. To consider the context of features we do the following:

$\square$ Selected metrics consider more than one feature class at a time. For instance, the feature classes $x$-dimension and $y$-dimension, i.e. the feature classes area and aspect ratio are considered together. Figure 5 shows an example of considering these classes.

$\square$ To express facts like "A n-input multiplexer can be constructed of two n/2 multiplexer and one 2-input multiplexer" we propose substitution rules which cause new search processes in addition to the current search. Substitution rules are represented as follows: 


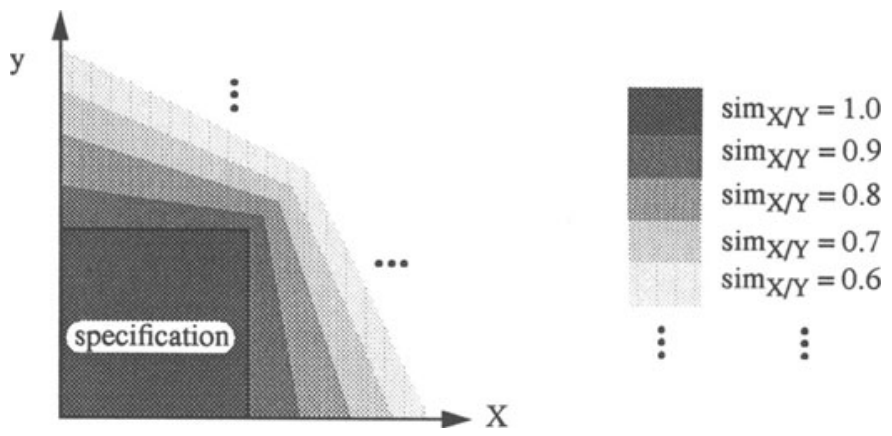

Figure 5: Considering the Two Feature Classes X-Dimension and Y-Dimension at a Given Specification

$$
\mathrm{P} \rightarrow \mathrm{P}_{1} \& \mathrm{P}_{2} \& \ldots \& \mathrm{P}_{\mathrm{n}} .
$$

If we search for a design object using a specification $s$ with the feature set $P_{s}$, the rule fires if the LHS $\mathrm{P}$ matches $\mathrm{P}_{\mathrm{S}}$ with $\mathrm{P} \subseteq \mathrm{P}_{\mathrm{S}}$. Then, $\mathrm{n}$ new search processes are started with new specifications $s_{i}, i=1, \ldots, n$, with:

$$
P_{s_{i}}=\left(P_{s}-\{P\}\right) \cup P_{i}
$$

The overall retrieval yield a result if either the old search process finds a suitable object or all new search processes caused by the RHS yield a result. The following example motivates this strategy:

\section{Example 3 (substitution rule)}

If we search for a multiplier with the specification ['Function is Multiply', 'Width is 16', 'Arity is 2', 'Technology CMOS'\} and we apply the substitution rule (see also figure 6):

\{'Function is Multiply', 'Width is n', 'Arity is 2'\} $\rightarrow$

\{'Function is Add', 'Width is n', 'Arity is 2'\} \&

\{'Function is Store', 'Width is n', 'Arity is 1'\} \&

\{'Function is Shift-Right', 'Function is Store',

'Width is $2 \mathrm{n}$ ', 'Arity is 1 ' $\}$;

then three new search processes are started with the specifications $s_{1}=$ ' 'Function is Add', 'Width is 16', 'Arity is 2', 'Technology CMOS' $\}, s_{2}=\{$ 'Function is Store', 'Width is 16 ', 'Arity is 2', 'Tech- 
nology CMOS' $\}$ and $s_{3}=\{$ 'Function is Shift-Right', 'Function is Store', 'Width is 32', 'Arity is 2', 'Technology CMOS'\}.

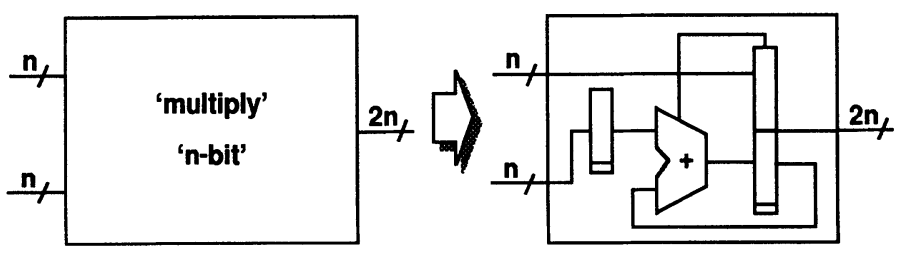

Figure 6: Substitution of a Multiplier by Network of other Modules

With these substitution rules, we exploit the part-of hierarchy. Another problem is the consideration of the design history. Using special metrics on the design state we exploit that adaptations can easier be made in more abstract descriptions than in more specific descriptions of design objects (see figure 7).

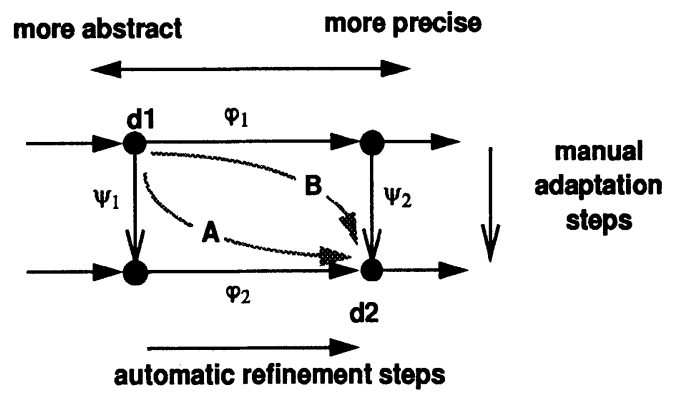

Figure 7: Use of the Design Hierarchy

It is obvious that, in this case, the way $A$ is easier to realize than the way $B$.

\section{Discussion}

The presented formalization is implemented in our prototype RODEO [AOS94]. (RODEO is an acronym for reuse of design objects.) As a part of the VLSI design system PLAYOUT [Zim89], RODEO supports designers in finding similar design objects and specifications. RODEO interactively delivers a set of reuse candidates which fit the current requirement specification best. RODEO has only an abstract view of the design data in accordance with the definitions 1 and 2 , i.e it does not consider detail data as netlists or layouts but only abstract features. It is necessary that the designer investigates the retrieved candidates in detail afterwards. Therefore, RODEO 
helps the designer and does not replace him.

Although the quality of our approach is attested by our implementation, our model does not consider all properties of section 2 . Therefore, we will finally gather the problems which must be solved toward a comprehensive reuse in ECAD: Firstly, we should minimize the checks by the designer after the retrieval process. Secondly, even if we use substitution rules the consideration of the feature context is not sufficient. It must be the goal to find context-sensitive descriptions of similarities. Thirdly, suitable domain-dependent metrics for the similarity function $\operatorname{sim}_{\mathrm{C}}$ for one or more feature classes must be determined. To solve this problem we must study many experiments with design reuse. Fourthly, even if RODEO contains an explantation component to support the designer using the retrieved design objects, more information should be gained towards an automatic adaptation step. And finally, the reuse of other design informations must be supported.

\section{References}

[AOS94] J. Altmeyer, S. Ohnsorge, B. Schurmann, "Reuse of Design Objects in CAD Frameworks", Proc. Int. Conference of Computer Aided Design, 1994

[BHK87] B. Becker, G. Hotz, R. Kolla, P. Molitor, "Hierarchical Design based on a Calculus of Nets", Proc. 24th Design Automation Conference, 1987

[CFI91] "Tool Encapsulation Specification", CFI Document No. 51, 1991

[DAC94] “Design Reuse: Fact or Fiction?", Panel, Proc. 31th Design Automation Conference, 1994

[GiC93] E. Girczyc, S. Carlson, "Increasing Design Quality and Engineering Productivity through Design Reuse", Proc. 30th Design Automation Conference, 1993

[Ger90] J. Gero, "Design Prototypes: A Knowledge Representation Schema for Design", AI Magazine, Winter, 1990

[HRS92] C. Huebel, D. Ruland, E. Siepmann, "On Modeling Integrated Design Environments", Proc. Int. European Design Automation Conference, 1992

[OnY87] M. Onosato, H. Yoshikawa, "A Framework on Formalization of Design Object for Intelligent CAD”, Proc. IFIP WG 5.2 Workshop on Intelligent CAD, Boston, 1987

[Sie91] E. Siepmann, "Entwurfstheorie und Entwurfsdatenmodellierung fuer CADFrameworks“, Ph.D. Dissertation, University of Kaiserslautern, 1991, (in German)

[Yos81] H. Yoshikawa, "General Design Theory and a CAD System", in "Man-Machine Communication in CAD/CAM", T. Sata, E. Warman (eds.), Proc. IFIP WG 5.2/5.3 Working Conference (Tokyo 1980), 1981

[Zim89] G. Zimmermann, "PLAYOUT - A Hierarchical Design System", Information Processing 89, G.X. Ritter (sd.), Elsevier Science Publishers B.V. (North Holland), IFIP, 1989 